\title{
Measurement Errors and Delays on Wide Area Control Based on IEEE Std C37.118.1-2011: Impact and Compensation
}

\author{
Lazaros Zacharia, Graduate Student Member, IEEE, Markos Asprou, Member, IEEE, and Elias \\ Kyriakides, Senior Member, IEEE
}

\begin{abstract}
Wide Area Control (WAC) of power systems is highly depended on synchronized measurements provided by the Phasor Measurement Units (PMUs). However, the contemporary measurement chain and the communication network are far from ideal. Therefore, it is essential to identify the impact of the availability and quality of the synchronized measurements, on the wide area controller's performance. This paper examines the effect of measurement errors and delays/dropouts on the damping capability of the wide area controller. The measurement errors considered in this work are according to the dynamic compliance requirements of the PMU during disturbances as it is imposed in the IEEE Synchrophasor Standard C37.118.1-2011. The delays are separated into measurement and feedback delays. The results indicate that delays along with dropouts deteriorate significantly the WAC performance, while the consideration of only the measurement errors (steady-state and dynamic) in the simulation environment has a minor effect on its operation. For this reason, a linear predictor is proposed to compensate effectively and timely all the system's delays. The case studies are conducted on the IEEE 39-bus system and evaluated through the Prony analysis tool.
\end{abstract}

Index Terms - IEEE Synchrophasor Standard C37.118.1-2011, linear predictor, measurement and feedback delays, measurement errors, wide area control.

\section{INTRODUCTION}

$\mathrm{T}$ HE electric power grid is a dynamic system which continually increases in size and complexity. A major challenge due to these changes, is the appearance of inter-area oscillations, which are highly undesirable, since they can degrade the power quality and can even deteriorate the power system's stability [1]. Inter-area oscillations occur when one group of generators starts to swing against the generators of another group. These oscillations are characterized by low frequencies $(0.1-1 \mathrm{~Hz})$ [2].

To eliminate the inter-area modes and furthermore, to increase the overall stability of the system, the concept of Wide Area Control (WAC) has emerged. The wide area controller aims to utilize the synchronized measurements in order to develop suitable coordination signals which enhance the

This work has been supported by the European Union's Horizon 2020 research and innovation programme under grant agreement No 739551 (KIOS $\mathrm{CoE}$ ) and from the Government of the Republic of Cyprus through the Directorate General for European Programmes, Coordination and Development. damping performance of the generators' local controllers in compensating effectively local and inter-area oscillations [3], [4]. The availability of synchronized measurements, through the Phasor Measurement Units (PMUs), is crucial for the WAC operation, since the observation of inter-area oscillations is only possible through their utilization. However, just like other realtime monitoring and control applications [5], WAC can be fully and successfully performed when accurate and timely measurements are provided by the measurement system.

Although PMUs are offering significantly higher accuracy compared to the conventional measurement devices, they are still prone to precision errors mainly due to the uncertainty introduced by the measurement chain and more specifically by the instrument transformers [6], [7]. Instrument transformers are utilized to provide isolation from the high-voltages and to step down the high voltage and current levels, to a level compatible with the measurement device that is connected to them [8]. In [7] a study took place considering the impact of the uncertainty of both measurement devices and instrument transformers on the accuracy of the Weighted Least Squares (WLS) state estimator. It has been illustrated that by obtaining weights of the measurements, based on the uncertainty of the whole measurement chain, the performance of the state estimator can be improved considerably. Furthermore, [9] and [10] present methodologies for the instrument transformer calibration through the estimation of the unknown ratio errors according to synchronized measurements. The authors in [11] investigated the improvement in the accuracy of the phasor measurements, when optical instrument transformers are considered instead of the traditional ones. Lastly, methods for the evaluation of the PMU measurement uncertainty are described in [12].

It is important to mention that for testing methodologies that are intended for dynamic conditions (such as the WAC that is based on PMUs), the PMU steady-state measurement errors are commonly considered, which is not completely in line with reality (e.g., [13]). More specifically, the PMU measurement errors increase significantly during dynamic conditions as it is

The authors are with the Department of Electrical and Computer Engineering and the KIOS Research and Innovation Center of Excellence, University of Cyprus, Nicosia, 1678, Cyprus (email: zacharia.lazaros@ucy.ac.cy; asprou.markos@ucy.ac.cy; elias@ucy.ac.cy). 


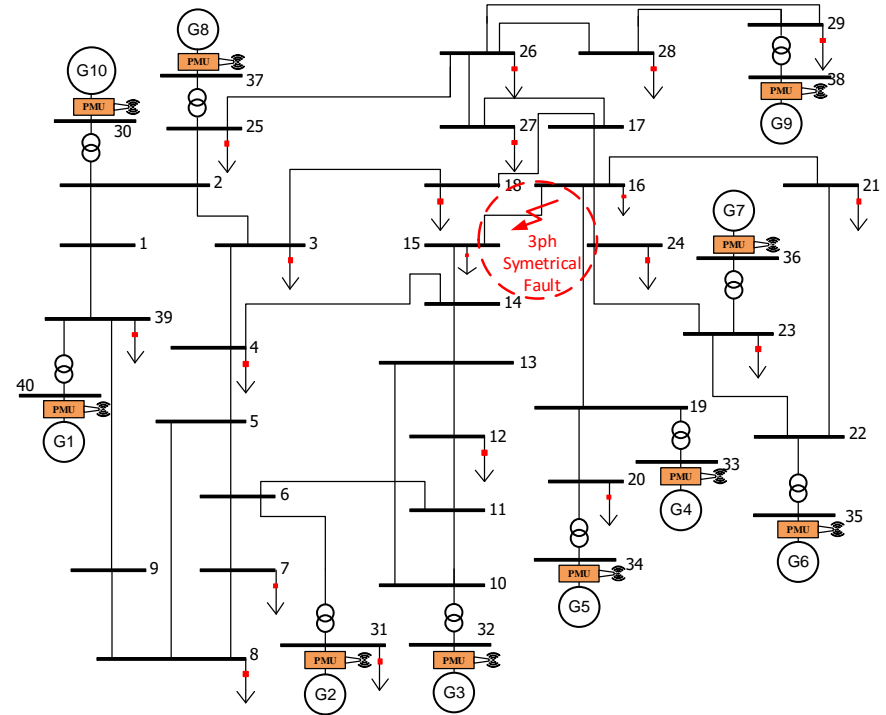

Fig. 1. IEEE 39-bus test system illustrating the considered fault.

reported in the IEEE Synchrophasor Standard C37.118.1-2011 [14]. This Standard is an updated version of the IEEE Synchrophasor Standard C37.118-2005 in order to incorporate the PMU dynamic compliance requirements as well. Furthermore, as it is clearly stated in [15], there is a need to investigate thoroughly the robustness of various synchrophasor applications to data quality issues, such as the data accuracy.

Apart from the measurement errors (which affect the precision of the synchronized measurements), another issue for the implementation of real-time applications is the fact that the communication networks are far from ideal. Generally, communication networks are characterized by data delays and dropouts [16]. The former occur during data exchange in a shared medium, while the latter can appear due to the noise in the communication lines [17]. In addition to the communication delays, the operational delays caused by the parts composing the real-time applications, are also significant [18]. Various studies have shown that the wide area controller's performance is vulnerable to the existence of data delays and data dropouts. In [19] a methodology to compensate the data dropout is presented, based on the Observer-driven Reduced Copy (ORC) approach in order to improve the performance of the wide area controller. Furthermore, in [20] a Smith predictor was utilized to preserve the performance of the controller within specific limits during large delays. Nevertheless, the majority of the published papers on this matter either utilize a simplified expression of the measurement errors or they do not consider them at all.

Therefore the main contributions of this paper are: (1) The consideration of the measurement errors of both instrument transformers and PMUs according to the IEC 61869-2/3 (for instrument transformers) and IEEE C37.118.1-2011 (for PMUs) for evaluating the performance of the WAC under dynamic conditions; (2) The investigation of the effect of measurement delays (in the presence of measurement errors), feedback delays and data dropouts due to the measurement and communication infrastructure on the WAC operation; and (3) The use of an efficient linear predictor that is first applied to
WAC for compensating measurement delays, feedback delays and data dropouts. Regarding the first contribution, to the authors best knowledge, this is the first work that considers the measurement errors of the PMUs during dynamic conditions (according to the IEEE C37.118.1-2011). Electro-Magnetic Transient (EMT) simulations on the IEEE 39-bus test system (Fig. 1) are used to examine the behavior of the wide area controller, while the Prony analysis tool is utilized to assess the damping performance of WAC for each case study.

The rest of the paper is organized as follows. Section II discusses the required wide area measurements and briefly presents the methodology for the development of the WAC signals. The effect of the instrument transformers and the PMU measurement errors on the WAC damping performance is presented in Section III. In addition, Section IV shows the respective results of the WAC when measurement and feedback delays are considered in the simulation along with dropouts and the achievable improvement of the controller performance when an advanced linear predictor is proposed and implemented. Finally, conclusions are provided in Section V.

\section{WidE AREA CONTROL Formulation}

In this section, the wide area measurements required for the formulation of the WAC and the development of the latter are presented. The wide area controller utilized in this study, is based on the hierarchical controller proposed in [21]. This methodology develops a wide area controller based on synchronized measurements and the reformulation of the system into a suitable and closed form.

\section{A. Wide Area Measurements}

The PMUs (like any other measurement device) are connected to the instrument transformers as shown in Fig. 2, which represents a simplified measurement chain [22]. Therefore, the accuracy of the synchronized measurements is highly depended on the precision of the instrument transformers. In this study, it is assumed that PMUs are installed at all the generator buses (Fig. 1) in order to provide all the necessary wide area measurements and the overall error of the measurement chain will be considered to assess the WAC damping capability.

The wide area (synchronized) measurements, which are required in order to apply the methodology of [21] are the generators' terminal voltage magnitudes $\left(v_{t}\right)$, frequencies $(f)$ and rotor angles $(\delta)$. The first two can be provided by a PMU.

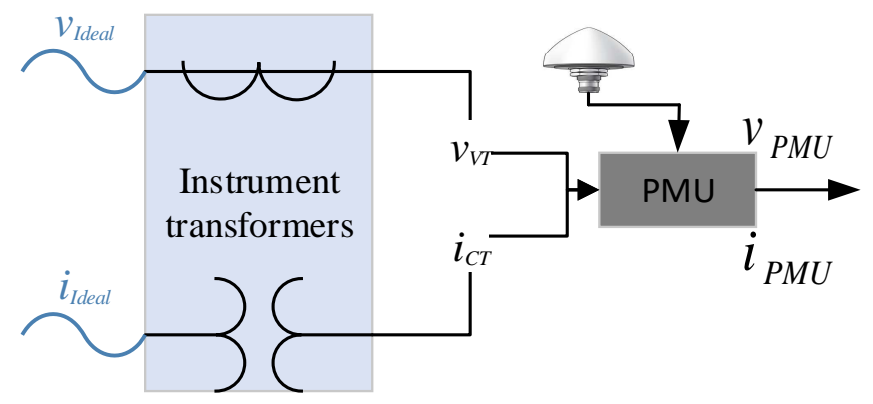

Fig. 2. Schematic diagram of the measurement chain, illustrating the connection of the voltage (VT) and current (CT) transformers with the PMU. 


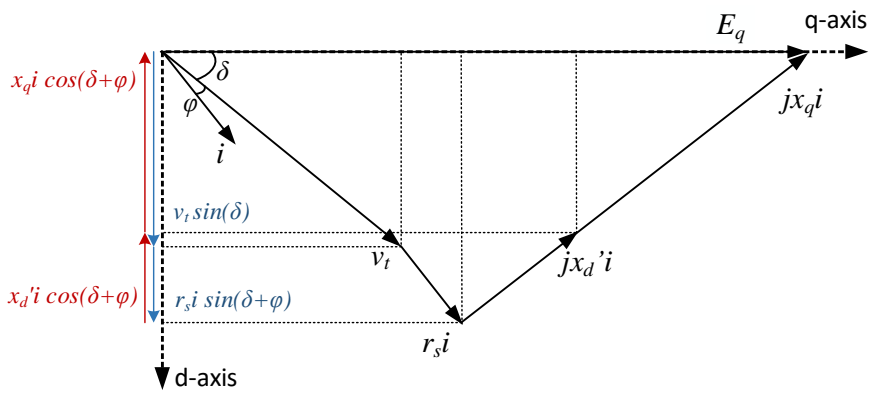

Fig. 3. Phasor diagram of a synchronous generator under transient conditions, without including a damper winding.

In [21], the rotor angle is assumed to be known. However, this is not the case in reality, as most of the commercial PMUs cannot measure the rotor angle directly. Therefore, in this work $\delta$ is estimated based solely on synchronized measurements. This step is needed in order to derive all the WAC inputs from the simplified measurement chain of Fig. 2.

For this purpose, the synchronous generator phasor diagram of Fig. 3 is utilized [23]. More specifically, it illustrates the behavior of the generator under transient conditions, neglecting only the damper winding (note that the stator resistance is included as well). By utilizing the phasor diagram, one can derive an expression of the rotor angle based on known quantities. This is achieved from the decomposition of all the vectors into the $d$ - $q$ axes. By considering only the $d$-axis, the next equation results:

$$
v_{t} \sin (\delta)=-r_{s} i \sin (\delta+\varphi)+\left(x_{d}^{\prime}+x_{q}\right) i \cos (\delta+\varphi)
$$

where $i$ represents the terminal current of the generator and $\varphi$ is the difference between the phase angle measurements of voltage and current.

Using the trigonometric identities for sine and cosine in (1), the following expression is derived for the rotor angle with respect to the terminal voltage magnitude of the generator:

$$
\tan (\delta)=\frac{\left(x_{d}^{\prime}+x_{q}\right) i \cos (\varphi)-r_{s} i \sin (\varphi)}{v_{t}+\left(x_{d}^{\prime}+x_{q}\right) i \sin (\varphi)+r_{s} i \cos (\varphi)}
$$

Looking at (2), one can realize that by knowing the generator parameters $\left(x_{q}, x_{d}^{\prime}\right.$ and $\left.r_{s}\right)$, the rotor angle can be estimated only from PMU measurements. Therefore, by considering the rotor angle estimation, the synchronized measurements required by the wide area controller are now the generator's terminal voltage (magnitude and angle), its terminal current (magnitude and angle), and its frequency.

\section{B. Development of Wide Area Signals}

The main idea of the methodology in [21] is firstly to reformulate the power system into a closed form and then to perform a change of variables in order to obtain a new state space representation of the power system. These new dynamic equations will have as state variables the terminal voltages of the generators, expressed in their local $d-q$ frame $\left(v_{d}\right.$ and $\left.v_{q}\right)$. To acquire this kind of expressions, the generator's fourth order model is utilized (as suggested in [24]), along with the stator dynamic equations. The former illustrates the relationship between the internal voltages $\left(e_{d}\right.$ and $\left.e_{q}\right)$, the stator currents $\left(i_{d}\right.$ and $\left.i_{q}\right)$ and the field voltage $\left(e_{f d}\right)$, as shown in (3)-(4). The latter represents the connection of the terminal voltages with the internal voltages and the stator currents ((5)-(6)).

$$
\begin{gathered}
T_{d o i}^{\prime} \dot{e}_{q i}^{\prime}=-e_{q i}^{\prime}-\left(x_{d i}-x_{d i}^{\prime}\right) i_{d i}+e_{f d i} \\
T_{q o i}^{\prime} \dot{e}_{d i}^{\prime}=-e_{d i}^{\prime}+\left(x_{q i}-x_{q i}^{\prime}\right) i_{q i} \\
v_{d i}=e_{d i}^{\prime}-r_{s i} i_{d i}+x_{q i}^{\prime} i_{q i} \\
v_{q i}=e_{q i}^{\prime}-r_{s i} i_{q i}-x_{d i}^{\prime} i_{d i}
\end{gathered}
$$

where $T_{d o}^{\prime}$ and $T_{q o}^{\prime}$ represent the open circuit time constants, while $x_{d}, x_{q}, x_{d}^{\prime}$ and $x_{q}^{\prime}$ are the generator's synchronous and transient reactances, all expressed in the local $d-q$ frame. Furthermore, $r_{s}$ is the stator resistance. All the quantities are in per unit, except the time constants which are in seconds.

The procedure to derive the new dynamic equations can be summarized in three steps. The goal is to utilize the new state space representation, in order to develop suitable coordination signals, able to increase the damping performance of the local controllers. For more information, the methodology has been fully explained in [21].

1) Voltages of the non-generator buses are expressed in terms of the generator bus voltages.

2) The internal voltage and stator current of each generator are expressed in terms of its terminal voltage.

3) The derived expressions are substituted in (3)-(4).

The result is the following new state space representation of the $i^{\text {th }}$ generator:

$$
\begin{aligned}
& \dot{v}_{d i}=a_{1 i} v_{d i}+a_{2 i} v_{q i}+p_{1 i} e_{f d i}+\psi_{d i} \\
& \dot{v}_{q i}=b_{1 i} v_{d i}+b_{2 i} v_{q i}+p_{2 i} e_{f d i}+\psi_{q i}
\end{aligned}
$$

where $a_{1}, a_{2}, b_{1}, b_{2}, p_{1}$ and $p_{2}$ are parameters which actually depend on the elements of the admittance matrix and the parameters of the generators. Furthermore, $\psi_{d}$ and $\psi_{q}$ represent the coupling perturbation terms (or inter-generator interactions) from other generators on the $i^{\text {th }}$ generator. Detailed expressions of all these parameters are included in [21].

The WAC signals can be derived based on the new dynamic equations now, by firstly replacing the $v_{d}$ and $v_{q}$ variables in (7) and (8), with their difference compared to their steady-state values $\left(d v_{d}\right.$ and $\left.d v_{q}\right)$ and by decomposing the excitation signal $e_{f d}$ into a local and a global part. The coordination signals are then obtained by choosing the global signal to explicitly cancel out all the inter-generator interactions $\left(\psi_{d}, \psi_{q}\right)$. This results to the following expression for the exciter coordination:

$$
e_{f d i}^{g}=\frac{-\left(\psi_{d v_{d}}^{i} d v_{d i}+\psi_{d v_{q}}^{i} d v_{q i}\right)}{p_{1 i} d v_{d i}+p_{2 i} d v_{q i}}
$$

\section{THE EFFECT OF INSTRUMENT TRANSFORMER AND PMU ERRORS}

This section analyzes the errors of the measurement chain considered in this study, as well as the simulation results of the WAC performance in the presence of these errors. 


\section{A. Measurement Errors}

As illustrated in Fig. 2, the measured quantities pass firstly through the instrument transformers and then through the PMU. Therefore, the errors affecting the WAC input signals are separated into the ones introduced by the sensing elements and the ones from the measurement devices. According to [7], these errors occur either due to random effects or due to systematic effects (existence of a constant "uncertainty" source). In this study, it is assumed that instrument transformers are properly calibrated to compensate all sources of systematic errors [22].

According to [11], the overall measurement error can be taken as the aggregation of the individual errors from the instrument transformers and the PMU, since the measurements of the former are transferred directly to the latter. In the case that no information about the probability distribution of the errors is available, a uniform distribution with the maximum errors (provided by the manufacturer) as upper and lower limits can be used [12]. As a result, the following expressions for the actual measurements provided by the PMUs, in terms of their ideal network values can be derived as:

$$
\begin{gathered}
v_{P M U}=v_{\text {Ideal }}\left(1+e_{P M U}^{v}\right)\left(1+e_{V T}^{v}\right) \\
\theta_{P M U}^{v}=\theta_{\text {Ideal }}^{v}+e_{P M U}^{\theta_{V}}+e_{V T}^{\theta_{V}} \\
i_{P M U}=i_{\text {Ideal }}\left(1+e_{P M U}^{i}\right)\left(1+e_{C T}^{i}\right) \\
\theta_{P M U}^{j}=\theta_{\text {Ideal }}^{j}+e_{P M U}^{\theta_{i}}+e_{C T}^{\theta_{i}} \\
f_{P M U}=f_{\text {Ideal }}+e_{P M U}^{f}
\end{gathered}
$$

where $e_{P M U}, e_{V T}$ and $e_{C T}$ stand for the measurement errors of the $\mathrm{PMU}$, the voltage transformer (VT) and the current transformer (CT) respectively. The angle $\theta$ is the phase of the measured quantity. Equations (10) and (12) reflect the magnitude of the measured quantities, while (11) and (13) their phase. Note that the error in $\delta$ results from the propagation of all the measurement errors of (10)-(13) through (2).

Table I and Table II summarize all the data regarding the maximum measurement errors of the instrument transformers and the PMUs respectively, which are considered as the maximum limits of the uniform distributions utilized to simulate the errors in (10)-(14). Without any loss of generality, the instrument transformers considered in this study, have an accuracy class of 0.5 . Note also that the current transformer maximum error changes according to the loading conditions of the transformer, with respect to its rated current. In Table I, the maximum measurement errors at $100 \%$ loading are used. The measurement errors utilized for the instrument transformers are all according to the IEC 61869-2 [25] for the CTs and the IEC 61869-3 [26] for the VTs. Furthermore, it is worth mentioning that the PMU data of Table II which correspond to steady-state conditions (as reported in the data sheet of [27] and in [7]), comply with the steady-state requirements of [14].

However, when dynamic conditions take place in the power system (e.g., due to a fault), the steady-state PMU measurement errors cease to be valid. For this reason, the dynamic
TABLE I

INSTRUMENT TRANSFORMER MAXIMUM ERRORS

\begin{tabular}{|c|c|c|c|c|c|}
\hline Type & $\begin{array}{c}\text { Accuracy } \\
\text { Class }\end{array}$ & \multicolumn{2}{|c|}{$\begin{array}{c}\text { Max Magnitude } \\
\text { Error (\%) }\end{array}$} & \multicolumn{2}{|c|}{$\begin{array}{c}\text { Max Phase Error } \\
\text { (degrees) }\end{array}$} \\
\hline $\mathrm{VT}$ & 0.5 & $e_{V T}^{v \max }$ & \pm 0.5 & $e_{V T}^{\theta_{V} \max }$ & \pm 0.333 \\
\hline $\mathrm{CT}$ & 0.5 & $e_{C T}^{i \max }$ & \pm 0.5 & $e_{C T}^{\theta_{i}}$ & \pm 0.5 \\
\hline
\end{tabular}

TABLE II

PMU STEADY STATE MAXIMUM ERRORS ACCORDING TO [27], [7]

\begin{tabular}{|c|c|c|c|c|c|c|c|}
\hline \multicolumn{2}{|c|}{$\begin{array}{c}\text { Max Voltage } \\
\text { Magnitude (\%) }\end{array}$} & \multicolumn{2}{|c|}{$\begin{array}{c}\text { Max Current } \\
\text { Magnitude (\%) }\end{array}$} & \multicolumn{2}{|c|}{$\begin{array}{c}\text { Max Phase Angle } \\
\text { (degrees) }\end{array}$} & $\begin{array}{c}\text { Max Frequency } \\
(\mathbf{H z})\end{array}$ \\
\hline$e_{P M U}^{v \max }$ & \pm 0.02 & $e_{P M U}^{i \max }$ & \pm 0.03 & $e_{P M U}^{\theta \max }$ & \pm 0.57 & $e_{P M U}^{f \max }$ & \pm 0.005 \\
\hline
\end{tabular}

TABLE III

PMU MAXIMUM ERRORS UNDER DYNAMIC CONDITIONS ACCORDING TO

\begin{tabular}{|c|c|c|c|c|c|}
\hline $\begin{array}{c}\text { Signal } \\
\text { Type }\end{array}$ & $\begin{array}{c}\text { Max } \\
\text { TVE } \\
(\boldsymbol{\%})\end{array}$ & $\begin{array}{c}\text { Magnitude } \\
(\boldsymbol{\%})\end{array}$ & $\begin{array}{c}\text { Phase } \\
\text { Angle } \\
(\mathbf{d e g r e e s})\end{array}$ & $\begin{array}{c}\text { Frequency } \\
(\mathbf{H z})\end{array}$ & $\begin{array}{c}\text { Time } \\
\text { Interval } \\
(\mathbf{s})\end{array}$ \\
\hline \multirow{2}{*}{ Voltage } & 8 & \pm 5.4 & \pm 3.3 & \pm 0.3 & 0.07 \\
\cline { 2 - 6 } & 12 & \pm 6.2 & \pm 4.58 & \pm 0.3 & 0.07 \\
\hline Current & 3 & \pm 1.6 & \pm 1.44255 & \pm 0.3 & 3 \\
\hline
\end{tabular}

compliance requirements of the IEEE Synchrophasor Standard C37.118.1-2011 [14] are utilized in this study in order to identify the appropriate dynamic measurement errors to be considered, as it will be shown in the next subsection. In [14] the dynamic compliance guidelines are provided for two performance class PMUs: the P-class (intended for protection applications) and the M-class (intended for analytic measurement applications). WAC requires the existence of the latter class in the system and therefore, the specifications for the M-class PMUs are considered in this study. These requirements include the limits of the Total Vector Error (TVE), the frequency error (FE), and the rate of change of frequency error (RFE). Note that TVE is a quantity which comprises both the amplitude and phase errors as specified in [14].

\section{B. Effect of Measurement Errors on WAC Performance}

The impact of the measurement errors due to the simplified measurement chain of Fig. 2 on the WAC operation are evaluated through simulations. For this reason, the IEEE 39-bus dynamic test system (Fig. 1) has been used, where generators are equipped with their own local controllers (exciter and governor). More specifically, the exciter DC2A and the general purpose governor are adopted. The test system and the WAC are constructed in MATLAB/Simulink, where discrete time EMT simulations are executed.

The objective of this section is to assess the damping capability of the WAC in the presence of measurement errors. This can be achieved through the comparison of the WAC performance with and without measurement errors (the case without measurement errors is hereafter referred as "ideal damping"). For the successful operation of the wide area controller, both local and inter-area oscillations should be compensated. Therefore, the terminal voltage of the generators is utilized as the local signal, while the speed difference between two distant generators is considered to follow the interarea oscillation damping. In this case study, a three-phase 


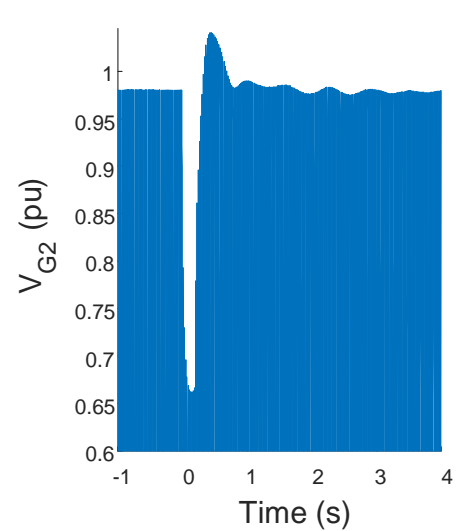

(a)

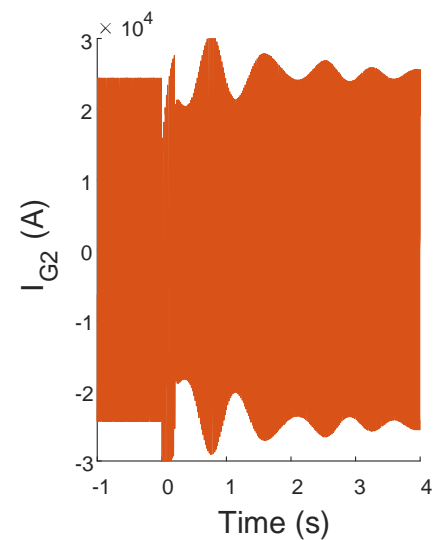

(b)
Fig. 4. Measurement signals from G2 during a fault: (a) voltage measurements and (b) current measurements.

grounded fault is assumed to occur on the line connecting bus 15 to bus 16 , at $\mathrm{t}=0 \mathrm{~s}$, as shown in Fig. 1 .

To consider the appropriate dynamic measurement errors in the simulation it is important to visualize the measurement signals received by the PMUs, during the disturbance. This step is necessary in order to identify which one amongst the three dynamic compliance tests (reported in [14]) is closer to their shape and therefore utilize the respective dynamic compliance requirements in the simulation. Since it is assumed that PMUs are located at all the generator buses, the measurement signals in this study are the generators' voltages and currents. Fig. 4 presents the voltage and current of generator 2 (G2) during the fault. Note that the measured voltages and currents from all the generators of the system have a similar form to the ones of G2. Based on Fig. 4, it can be seen that the voltage behaves like a stepped signal after the occurrence of the fault, while the current can be viewed as a modulation signal. Therefore, the dynamic compliance requirements for the input step change and the modulation signal tests are utilized as the dynamic measurement errors of all the voltage and current measurements, respectively. Table III presents the PMU maximum errors under dynamic conditions, according to the amendment of the IEEE Synchrophasor Standard C37.118.12011 [28]. Note that the time interval to return back to the steady-state errors (after the disturbance) is found through the response time requirement of [28] for the input step change test. In the case of the modulation signal test, the respective time interval is not defined in the Standard and therefore, it is taken as the duration until the magnitude deviation of the measured signal (compared to the pre-fault signal) is lower than $0.1 \mathrm{pu}$. Regarding the TVE of the input step change test, the IEEE Synchrophasor Standard C37.118.1-2011 states that for a 10\% change in the magnitude the TVE reaches $6 \%$. Therefore, in this work since the voltage magnitude change is larger than $10 \%$ (Fig. 4a), both $8 \%$ and $12 \%$ TVEs are considered.

The WAC performance evaluation is carried out by utilizing the Prony analysis, which provides all the information for the power system stability. More specifically, Prony analysis is a measurement-based method which is used for ringdown analysis and for small-signal properties' extraction of damped signals [2]. It is worth mentioning that Prony method is extensively utilized in the reports of various well-established organizations for analyzing the appearance of inter-area modes in the system, such as the ENTSO-E [29]. The significant advantage of this methodology is that it does not require a model of the system and it provides accurate mode estimation since it is applied on the actual response of the system. The objective is to extract the damping ratio $(\zeta)$ and frequency $(f)$ of local and inter-area modes. A higher damping ratio means better WAC performance. Due to space limitations, only selected terminal voltages and speed differences from the IEEE 39 bus, will be utilized for the graphical illustrations.

Fig. 5 depicts the simulation results for the terminal voltage of G2 and the speed difference between G7 and G10 $\left(w_{7}-w_{10}\right)$. Actually, it represents a comparison of the system's response between the scenarios where no WAC exists in the system (No WAC), the ideal damping, when only the steady-state measurement errors (SSME) are considered, and the scenarios where the dynamic measurement errors (DME 1 and DME 2) occur during the disturbance (according to the data of Table III). Note that DME 1 corresponds to 8\% TVE while DME 2 corresponds to $12 \%$ TVE. In both scenarios, the TVE on the current phasor measurements is kept to $3 \%$. Based on Fig. 5, it

TABLE IV

PRONY ANALYSIS FOR THE MEASUREMENT ERRORS

\begin{tabular}{|c|c|c|c|c|}
\hline \multirow{2}{*}{ Type } & \multicolumn{2}{|c|}{ No WAC } & \multicolumn{2}{c|}{ Ideal Damping } \\
\cline { 2 - 5 } & $\zeta(\%)$ & $f(\mathrm{~Hz})$ & $\zeta(\%)$ & $f(\mathrm{~Hz})$ \\
\hline Local mode & 5.58 & 1.2 & 18.25 & 1.2 \\
\hline Inter-area mode & 29.9 & 0.71 & 38.3 & 0.69 \\
\hline \hline \multirow{2}{*}{ Type } & \multicolumn{2}{|c|}{ SSE } & \multicolumn{2}{c|}{ DME 1 } \\
\cline { 2 - 5 } & $\zeta(\%)$ & $f(\mathrm{~Hz})$ & $\zeta(\%)$ & $f(\mathrm{~Hz})$ \\
\hline Local mode & 17 & 1.2 & 15.88 & 1.2 \\
\hline Inter-area mode & 38.3 & 0.69 & 36.5 & 0.69 \\
\hline \hline Type & \multicolumn{3}{|c|}{ DME 2 } & \\
\cline { 2 - 3 } & $\zeta(\%)$ & $f(\mathrm{~Hz})$ & & \\
\hline Local mode & 15.88 & 1.2 & & \\
\hline Inter-area mode & 36.5 & 0.69 & & \\
\hline
\end{tabular}

(a)
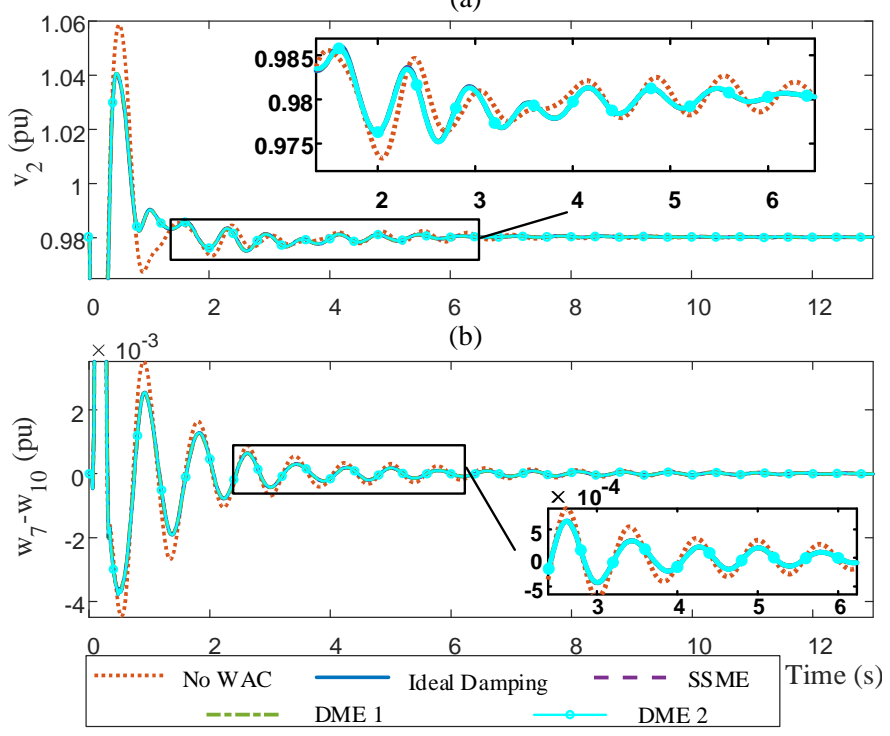

Fig. 5. Comparison of system's response between No WAC, Ideal Damping, WAC with steady-state measurement errors (SSME), WAC with dynamic measurement errors (DME 1) where TVE for voltage and current is equal to 8\% and 3\%, and WAC with dynamic measurement errors (DME 2) where TVE for voltage and current is equal to $12 \%$ and $3 \%$. (a) Local oscillation of the terminal voltage of G2 and (b) the inter area oscillation between G7 and G10. 


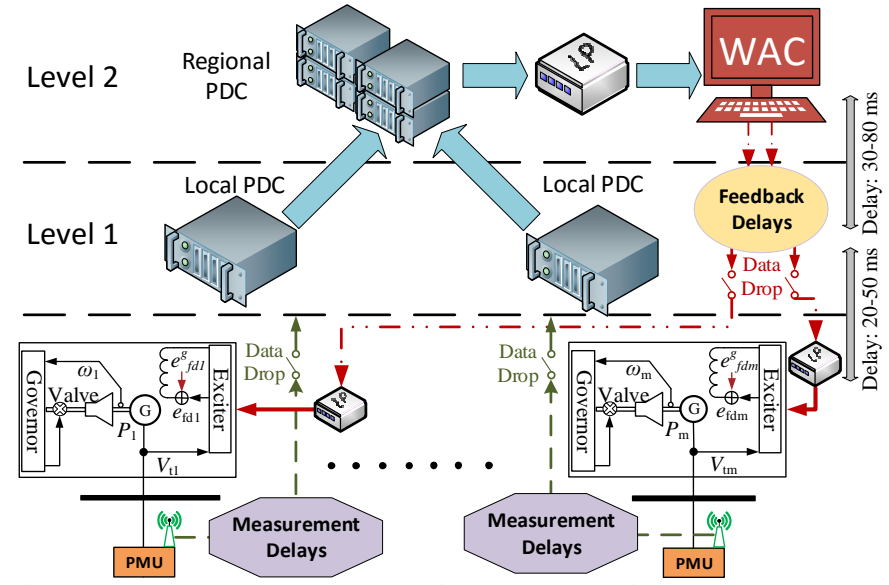

Fig. 6. Measurement delays included for the data transfer between the PMUs, the local PDCs (Level 1) and the regional PDC/WAC (Level 2), and feedback delays from the WAC to the local controllers.

can be seen that the overall measurement error introduced by the measurement chain (in the case of both the steady-state only and the appropriate dynamic PMU measurement errors during the fault) has negligible effect on the performance of the wide area controller in compensating local and inter-area oscillations. This result can be further supported by the Prony analysis results of Table IV, where it is evident that the damping ratio is extremely close for each case for all the respective modes. The outcome of this case study illustrates clearly that if all the necessary inputs are originated from PMUs, they can be used directly to the wide area controller without compromising the stability of the system. Therefore, the usage of a state estimator for filtering the measurement errors can be avoided (decreasing that way the overall application delays) and it should be considered for estimating only any unknown WAC inputs.

\section{IMPACT OF MEASUREMENT ERRORS AND DELAYS}

In this section the WAC behavior is studied when realistic measurement/feedback delays, dropouts and errors occur simultaneously. This is one of the contributions of this work as the usual case is to test the wide area controller methodologies under the event of data delays/dropouts only or by combining them with steady-state PMU errors. In addition, a linear predictor is proposed in the examined scenario, in order to illustrate the improvement on the wide area controller's performance, when the measurement/ feedback delays and data dropouts are effectively compensated.

\section{A. Measurement/Feedback Delays and Data Dropouts}

Measurement delays occur due to the data processing and transfer of measurements and they are generally known to be the main reason for the degradation of the WAC damping capability, along with data dropouts [20], [3]. Data dropout is an unpredictable phenomenon, which results due to transmission errors/noise or buffer congestion [19]. As it was aforementioned in Section I, the majority of the works which investigate the effect of data delays/dropouts on WAC, are either utilizing a simplified steady-state expression for the measurement errors or they do not consider them at all.
However, in reality the data delays/dropouts take place along with the overall errors of the measurement chain (which change during disturbances). Therefore, the consideration of all of them in the simulation is essential for obtaining more realistic results.

In various studies, measurement delays are taken as constant or time-varying (e.g., [17]), but in reality they have a random nature due to the existence of diverse communication channels and data traffic. According to the IEEE Synchrophasor Standard C37.118.2-2011 the delays of the measurement infrastructure are defined as the interval from the time when a signal has a certain value until the time when the measurement is used by the application. Measurement delays are separated into the measurement process delays and the communication delays [30]. The measurement process delays include the sampling procedure, the filtering and the data processing/alignment delays. The communication delays are due to the distance, the medium type, the data buffering and the time required to send each bit.

Some available studies do not consider that there is also a delay on the feedback control signal (from the WAC to the local controllers) and therefore, they concentrate only on dealing with the delays between the PMUs and the wide area controller (e.g., [3] and [17]). However, especially the inclusion of the former is equally important since it can adversely impact the operation of WAC and thus it can jeopardize the stability of the whole system. In this study, delays in both directions (measurement and feedback delays) are taken into account for obtaining more realistic results, as it is illustrated in Fig. 6 . Since there is no measurement procedure into the path of the

TABLE V

EXAMINED CASES AND PDF PARAMETERS

\begin{tabular}{|c|c|c|}
\hline Case & Measurement Delay Model & Feedback Delay Model \\
\hline I & Uniform $(\max =250, \min =50)$ & Gamma $(x=30, y=3)$ \\
\hline II & Uniform $(\max =250, \min =50)$ & Beta $(c=18, d=188)^{*}$ \\
\hline
\end{tabular}

where $x$ and y are the shape and scale parameters of the Gamma PDF, while $c$ and $d$ represent the first and second shape parameter for the Beta PDF.

* Note that the Beta PDF is in seconds, while Uniform and Gamma are in ms.

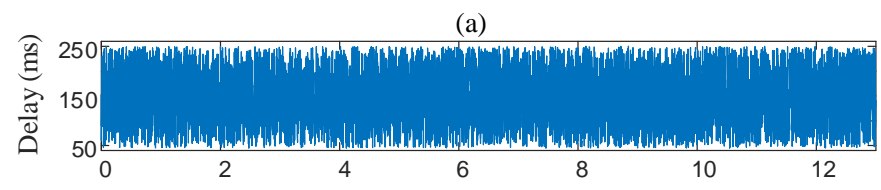

(b)

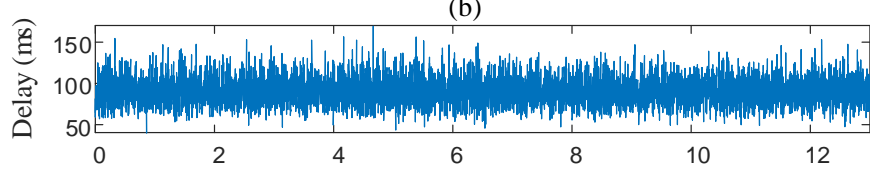

(c)

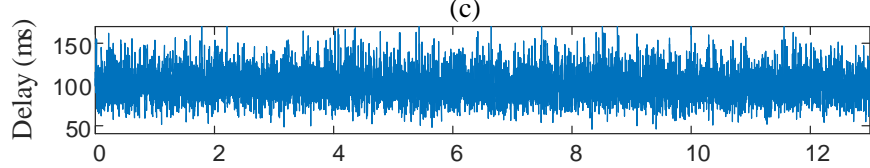

(d)

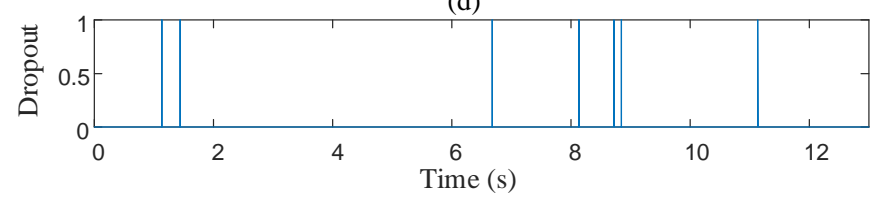

Fig. 7. (a) Uniform measurement delays, (b) Gamma feedback delays, (c) Betta feedback delays, and (d) data dropouts. 
feedback signal, a small Gaussian noise is considered along with the feedback delays to simulate the channel noise. Note here that the feedback delays consist of the application delays (processing of the end device and the application methods) and the communication delays that are associated to the transfer of the WAC signals back to the system. Since both, measurement and feedback delays utilize the communication infrastructure, data dropouts are considered to occur along with these delays.

For the simulation of the measurement delays and the feedback delays, combinations of three different Probability Distribution Functions (PDFs) are utilized in the delay models. The PDFs considered in this study are the uniform [31], Beta [32] and Gamma [33] distributions. More specifically, Table V summarizes the examined cases and the adopted parameters of the PDFs in each case. The uniform distribution is adopted for modelling the measurement delays, since various sources of delay exist in the communication path from the PMUs until the WAC. Beta and Gamma are used to model the feedback delays, as they are typical PDFs for simulating packet-based networks. Furthermore, it is important to mention that the range of the measurement delays utilized in this work, is [50-250] ms, while for the feedback delays is [40-160] ms. Note that Fig. 7 illustrates the measurement delays (for one PMU) and feedback delays (for one control signal), along with the data dropouts considered in the simulation.

Regarding the lower bound of the measurement delay range $(50 \mathrm{~ms})$, this was selected to be in line with the IEEE Synchrophasor Standard C37.118.2-2011. As it is reported in

(a)

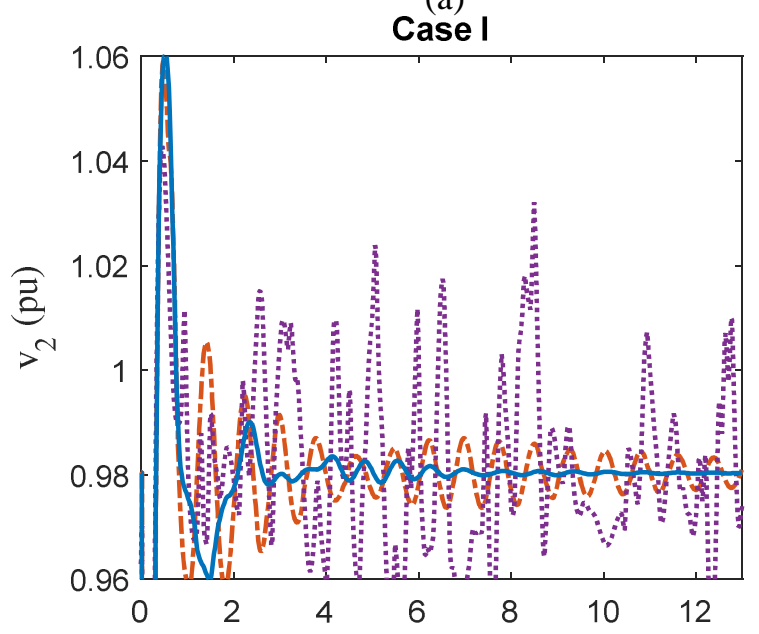

(c)

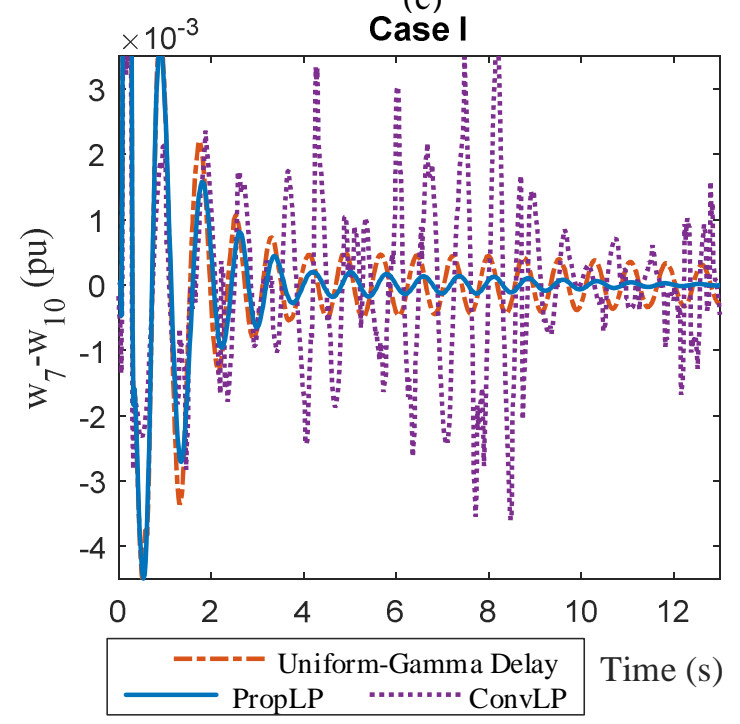

(b)

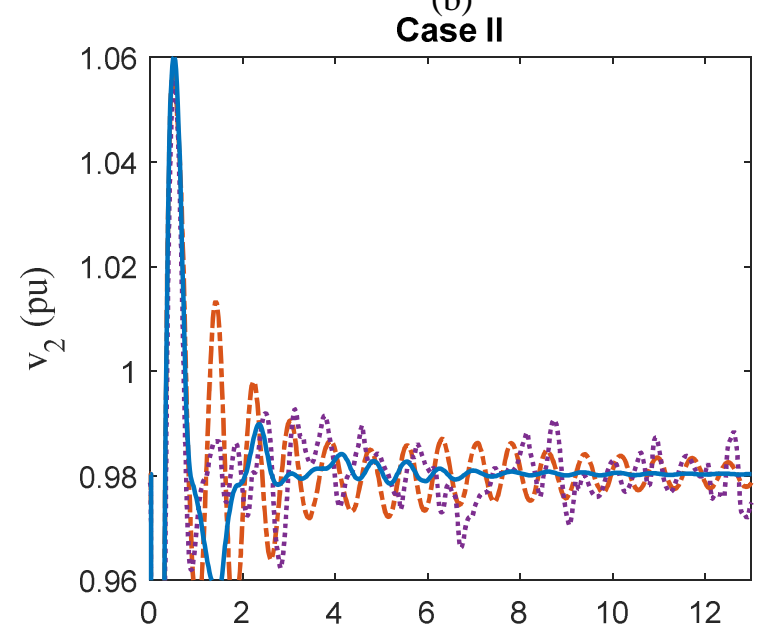

(d)

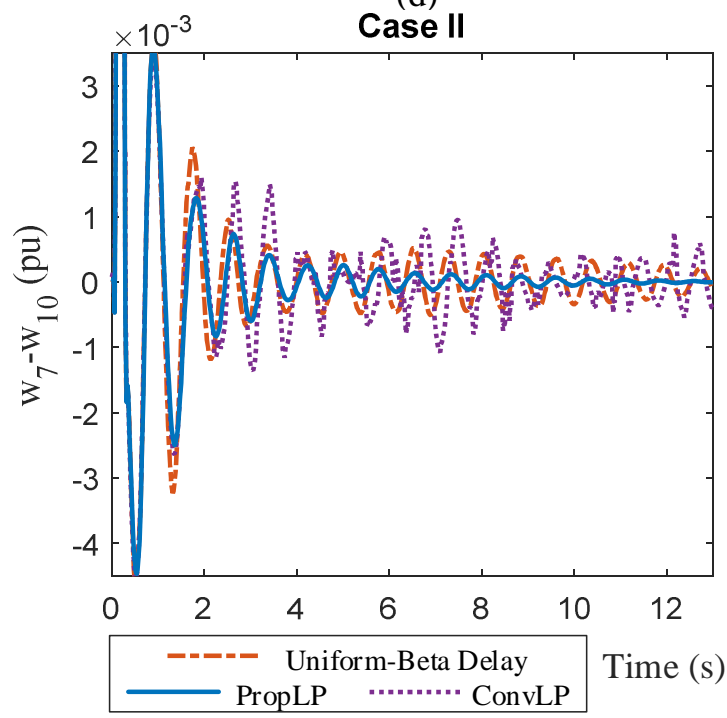

Fig. 8. Comparison of WAC performance for the delays of Case I and II, when no delay compensation exists and when the conventional and proposed predictors are utilized, considering: (a)-(b) the local oscillation of the terminal voltage of G2, and (c)-(d) the inter area oscillation between G7 and G10 for each case.

TABLE VI

PRONy ANALysis Results For the Data Delays of CASEs I-II, CONVENTIONAl LiNEAR PREDictor

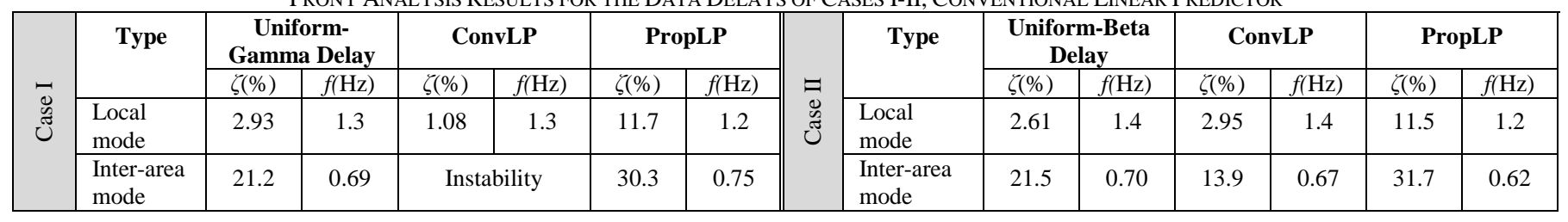


this standard, typical delays from a PMU to the Phasor Data Concentrator (PDC) are between 20-50 ms and each level above adds additional delays in the range of 30-80 ms [30]. Therefore, as it is depicted in Fig. 6, the simulation considers local PDCs on the first level and a regional PDC to the WAC application on the second level. The upper bound of the considered range is selected to be $250 \mathrm{~ms}$, according to the relatively high delays reported in [34]. Note that the minimum delay of $50 \mathrm{~ms}$ is also in line with the measurement reporting latency compliance of [14] which is 5/(PMU reporting rate) for the M class PMUs. In this work, the reporting rate is 100 phasors/second.

As it was aforementioned, the PDC concept is considered in this study in order to forward the PMU data to the wide area controller. The reporting rate of the PDC can be selected based on the range of the measurement delays. More specifically, the minimum rate at which it can operate (in order to take into account the data delays of the measurements) is $50 \mathrm{~ms}$. The problem that arises here is that at $50 \mathrm{~ms}$ very few measurements will arrive to the PDC on time. Therefore, the "absolute wait time" concept has been applied in the PDC, as it is specified in the IEEE Synchrophasor Standard C37.244-2013 [35]. This represents the maximum time that various measurement-based tools (such as the PDC) can wait for the synchronized measurements to arrive after the expected timestamp [16]. If the "absolute wait time" expires, the PDC utilizes the previously received measurement and it discards any delayed data received afterwards. In this way the packet disorder is avoided. The time interval that the PDC can wait for the measurements to arrive is considered to be $20 \mathrm{~ms}$. This is an extra time added to the minimum reporting rate $(50 \mathrm{~ms})$. Therefore, the "wait time" (and thus the maximum reporting rate of the PDC) is equal to $70 \mathrm{~ms}$. In the case where all the measurements are received before the "wait time" expires, the PDC forwards them directly to the WAC.

In order to investigate the effect of the measurement and feedback delays on the WAC performance, the same test system and disturbance (as were described in Section III.B), are considered. Therefore, the same dynamic measurement errors are applied here too. In this sense, the measurements in this case are affected by measurement errors, delays and dropouts. The negative effect of the delays and dropouts on the WAC performance can be seen graphically and numerically in Fig. 8 and Table VI respectively. As shown, in all the examined cases, the data delays can impact negatively the damping capability of the wide area controller, leading the system even to instability. More specifically, the WAC is unable to damp the local and inter-area oscillations in the presence of delays/dropouts. Note that this is also evident when the local and inter-area damping ratios of Cases I-II are considered (Table V), which are lower than the No WAC scenario of Table IV. Therefore, the inclusion of a data delay compensator is essential for the WAC operation.

\section{B. Linear Predictor}

To compensate the measurement delays, it is a common practice to utilize predictors in the architecture, in order to restore timely any lost measurements. Various methodologies have been proposed to address this issue, such as the Smith
TABLE VII

COMPARISON OF PREDICTION METHODS

\begin{tabular}{|c|c|c|c|c|c|}
\hline $\begin{array}{l}\text { Prediction } \\
\text { Method }\end{array}$ & $\begin{array}{c}\text { Smith } \\
\text { Predictor } \\
\text { [20] }\end{array}$ & $\begin{array}{c}\text { MEKF } \\
\text { [36] }\end{array}$ & $\begin{array}{l}\text { Lead- } \\
\text { Lag } \\
\text { Filter } \\
{[37]}\end{array}$ & $\begin{array}{c}\text { Linear } \\
\text { Predictor } \\
\text { [38] }\end{array}$ & $\begin{array}{l}\text { Proposed } \\
\text { Predictor }\end{array}$ \\
\hline $\begin{array}{c}\text { Model Size } \\
\text { Independency }\end{array}$ & & & & & \\
\hline $\begin{array}{c}\text { Feedback } \\
\text { Control Delay } \\
\text { Compensation }\end{array}$ & & & & & \\
\hline $\begin{array}{c}\text { Data } \\
\text { Dropouts } \\
\text { Compensation }\end{array}$ & & & & & \\
\hline $\begin{array}{c}\text { Dynamic } \\
\text { Delay } \\
\text { Consideration }\end{array}$ & & & & & \\
\hline $\begin{array}{l}\text { Measurement } \\
\text { Error } \\
\text { Consideration }\end{array}$ & & & & & \\
\hline
\end{tabular}

Predictor [20], Extended Kalman Filter (EKF) [36], and Holt's linear exponential smoothing [6]. However, the performance/ accuracy of the majority of these methods is directly depended on the model of the system that they are using. The main drawback of such model-based methods is that when larger systems are considered, more detailed models are required and thus the complexity and processing time increases rapidly. Furthermore, as pointed out in [37] the commonly used Smith predictor and Pade approximation are effective methodologies for compensating constant delays only.

To overcome this issue, predictors which are based only on measured data are implemented. An example is shown in [37] where an adaptive time delay compensator is implemented based on several lead-lag filters (each one is appointed to a different time interval). As clearly stated in [37], this methodology suffers from various disadvantages. More specifically, it fails in the case of frequent delay changes or in the presence of large delays, which can lead the system to instability. Furthermore, the implementation of a prediction based hierarchical delay compensation (PHDC) method is presented in [38], which is developed by considering a Linear Prediction (LP). The utilization of LP is also adopted for increasing the simulation accuracy of distributed real-time simulators as shown in [39]. Although the measurement-based predictors of [37] and [38] show promising results, they both suffer from processing time issues (in larger systems) since they derive several predictions at each iteration for each feedback control signal and they select the appropriate one at the local controller's (actuator) level. In addition, both methodologies totally disregard the existence of data dropouts in the network which can even lead the system to instability.

Characteristics of some of the aforementioned model-based and measurement-based methodologies for data delays/dropout compensation are summarized in Table VII. Note that none of these methods considers the existence of measurement errors (which can potentially affect the prediction) and that only one includes the compensation of the data dropouts. A faster and less complex predictor is proposed by the authors in [40], which is based on Autocorrelation Linear Predictive Coding (LPC). The overall idea is that any future signal sample $\left(\hat{u}_{M+1}\right)$ can be 
approximated as a linear combination of its present $\left(u_{M}\right)$ and a finite number of past samples $\left(u_{i}\right)$. This can be achieved by determining the coefficients of the linear predictor $\left(a_{i}\right)$ in a way that they will minimize the prediction error in the least squares sense (16):

$$
\begin{gathered}
\hat{u}_{M+1}=-\left(a_{2} u_{M}\right)-\left(a_{3} u_{M-2}\right)-\ldots-\left(a_{N+1} u_{M-N+1}\right) \\
\min \|U \tilde{a}-b\|
\end{gathered}
$$

$$
\begin{gathered}
\text { where } \quad \tilde{a}=\left[a_{2} \ldots a_{N+1}\right]^{T} \\
U=\left[\begin{array}{ccccccccc}
u_{1} & u_{2} & \ldots & u_{i} & \ldots & u_{M} & 0 & \ldots & 0 \\
0 & u_{1} & u_{2} & \ldots & u_{i} & \ldots & \ldots & \ddots & \ddots \\
\vdots & \ddots & \ddots & \ldots & \ldots & \ldots & \ldots \\
0 & \ldots & 0 & u_{1} & \ldots & \ldots & \ldots & \ldots & 0 \\
0 & \ldots & u_{M}
\end{array}\right]^{T}
\end{gathered}
$$

Note that $N$ represents the size of the coefficient vector $\tilde{\alpha}$ and the window length which holds the present and a finite number of the past signal samples, required for the prediction. According to various tests on the IEEE 9 and 39 bus test systems, an $N=20$ is found sufficient for accurate predictions.

In this case study, a more advanced linear predictor is implemented to address the measurement/feedback delays effectively and timely. More specifically, the linear predictor presented in [40] requires the prediction error resulted from the previous prediction step to be added on the next prediction. This has as a result to keep the predicted value relatively close to the actual value of the measurement. However, this technique is especially effective when small delays occur $(\sim 20 \mathrm{~ms})$ and therefore the majority of the signals samples $u_{i}$ (which are used in the LPC process) are the actual values of the measured signal. Due to the fact that in this study large delays are considered; the $u$ vector will consist mostly of previous predictions. To acquire better and more accurate predictions in this case, the following expression has been utilized:

$$
\begin{gathered}
\hat{u}_{M+1}=-\left(a_{2} u_{M}\right)-\left(a_{3} u_{M-1}\right)-\ldots-\left(a_{N+1} u_{M-N+1}\right) \\
+\left(k_{2} P_{M}\right)+\left(k_{3} P_{M-1}\right)+\ldots+\left(k_{N+1} P_{M-N+1}\right)+e_{p r d} \\
k_{i}=\frac{-\Delta_{i-1}}{P_{i-1}} \\
e_{p r d}=\left|\hat{u}_{M}-u_{M}\right|
\end{gathered}
$$

where $k_{i}$ are the reflection coefficients which result from the Autocorrelation LPC procedure and $P_{i}$ stands for the prediction error power of each of the previous predictions. $\Delta_{i-1}$ is actually the cross-correlation of the forward prediction error and the unit delayed backward prediction error. $e_{p r d}$ represents the prediction error feedback, as it is introduced in [40]. Note that in the case where $u_{j}$ data arrive before the "wait time" expires (and therefore the predicted value is not utilized), the prediction error power $P_{j}=0$. It is important to mention here that whenever a new signal sample arrives (either the actual or the predicted
TABLE VIII

MEAN SQuARE ERRor OF THE DELAYED Signal AND THE Two LPS

\begin{tabular}{|c|c|c|c|}
\hline Measurement & No LP & ConvLP & PropLP \\
\hline $\boldsymbol{v}$ & 0.0036 & 0.9403 & 0.0025 \\
\hline $\boldsymbol{\delta}$ & 0.0084 & 0.3009 & 0.0074 \\
\hline
\end{tabular}

one) the LPC procedure estimates the new linear predictor coefficients $a_{i}$ and the reflection coefficients $k_{i}$ according to (16) and (18).

The proposed linear predictor (PropLP) is considered to compensate the impact of the data delays in Cases I and II (Fig. 8 and Table VI). To the authors best knowledge this is the first time that a linear predictor of this type is utilized in WAC applications. Note that the same PDFs and delay ranges (as described in the previous subsection) are considered here as well, along with data dropouts and measurement errors which are applied on the measured signals. Furthermore, it is worth mentioning that linear predictors are placed both on the wide area controller and on the generator local controllers (exciter) as shown in Fig. 6, to compensate effectively the measurement delays and the feedback delays, respectively.

Apart from the proposed linear predictor, a conventional LP (ConvLP) according to (15) is also considered into the simulations. This is done in order to present the significant improvement of the WAC performance when the proposed scheme is utilized. Fig. 8 and Table VI illustrate the simulation results for the compensation of the local and inter-area oscillations when the same three-phase grounded fault occurs. As shown, the inclusion of the proposed linear predictor in the WAC scheme improves considerably the damping performance of the system, compared to the case where the data delays/dropouts are left uncompensated. More specifically, the consideration of the proposed predictors in the simulation makes the wide area controller capable of bringing the system back to stability. Conversely, the utilization of the conventional LP is unable to compensate effectively the data delays/dropouts, leading the system to instability. This is also confirmed through the Prony analysis results of Table VI. Here, it can be seen that the WAC combination with the proposed linear predictor achieves high damping ratios, while the damping ratios drop significantly in the cases of the conventional predictor or when no delay compensation is considered at all.

Accuracy information regarding the approximation of the non-delayed signal for all the three examined cases (No LP, ConvLP and PropLP) are provided in Table VIII. In particular, the mean square error (MSE), calculated as shown in (20), is tabulated. As it is illustrated the MSE of the ConvLP is much larger than the other two cases. This is because the prediction error feedback $\left(e_{p r d}\right)$ is not considered at all in (15), in order to improve the future predictions. In contrast, as clearly shown in (17), the PropLP considers the prediction error power along with the prediction error feedback in the calculation of the future predictions.

$$
M S E=\frac{1}{L} \sum_{i=1}^{L}\left(Y_{i}-\tilde{Y}_{i}\right)^{2}
$$


where $L$ is the total number of the data points considered, $Y_{i}$ stands for the non-delayed signal and $\tilde{Y}_{i}$ represents either the delayed signal in the case where there is no delay compensation (No LP) or the compensated signal when a linear predictor is used (ConvLP/PropLP). All these results indicate both the effectiveness of the proposed linear predictor and the necessity of having an accurate linear predictor in the WAC architecture.

\section{CONCLUSION}

This paper presents an investigation regarding the WAC performance under measurement errors (of both, instrument transformers and PMUs) and data delays/dropouts combined with errors. The paper suggests the use of a proposed linear predictor to compensate all the delays/dropouts. The measurement errors considered in this study are implemented to comply with the dynamic compliance requirements of the IEEE Synchrophasor Standard C37.118.1-2011 in order to obtain realistic measurements during a disturbance. All the case studies are executed using the IEEE 39-bus test system under a three phase to ground fault using EMT analysis. As it can be concluded by the graphical and numerical simulation results, the measurement chain's errors do not have a severe impact on the WAC performance. Conversely, the appearance of measurement and feedback delays, along with data dropouts, can impact significantly the power system stability. More specifically, combinations of three different PDFs (uniform, Beta and Gamma) are considered to model the measurement and feedback delays. The results indicate that in the presence of data delays and dropouts the WAC damping performance is deteriorated. In order to compensate such delays, a linear predictor is proposed to be utilized in the WAC scheme, which increases significantly the damping capability of the wide area controller. By using the proposed linear predictor, the WAC becomes capable of bringing the system back to stability.

In conclusion, based on the results of this paper, in a system where all the required WAC inputs originate from PMUs, their direct use in the wide area controller after the alignment is recommended (reduce the application delays). This is because, data delays and dropouts are found to be the actual threat of the WAC operation and not the measurement errors. Furthermore, it is identified that the existence of an effective prediction scheme capable of compensating both the measurement and feedback delays is crucial for maintaining the system's stability.

\section{REFERENCES}

[1] M. Jonsson, M. Begovic, and J. Daalder, "A new method suitable for real-time generator coherency determination," IEEE Trans. Power Systems, vol. 19, no. 3, pp. 1473-1482, 2004.

[2] A. R. Messina, Inter-area Oscillations in Power Systems: A Nonlinear and Nonstationary Perspective (Power Electronics and Power Systems), Guadalajara: Springer, 2009.

[3] W. Yao, L. Jiang, J. Wen, S. J. Cheng, and Q. H. Wu, "Networked predictive control based wide-area supplementary damping controller of SVC with communication delays compensation," in IEEE PES General Meeting, Vancouver, 2013.

[4] M. Beiraghi and A. M. Ranjbar, "Additive model decision tree-based adaptive wide-area damping controller design," IEEE Systems Journal, vol. 12, no. 1, pp. 328-339, 2018.
[5] M. K. Jena, S. R. Samantaray, and B. K. Panigrahi, "A new wide-area backup protection scheme for series-compensated transmission system," IEEE Systems Journal, vol. 11, no. 3, pp. 1877-1887, 2017.

[6] F. Aminifar, M. Shahidehpour, M. Fotuhi-Firuzabad, and S. Kamalinia, "Power system dynamic state estimation with synchronized phasor measurements," IEEE Trans. Instrum. Meas., vol. 63, no. 2, pp. 352-363, 2014.

[7] M. Asprou, E. Kyriakides, and M. Albu, "The effect of variable weights in a WLS state estimator considering instrument transformer uncertainties," IEEE Trans. Instrum. Meas., vol. 63, no. 6, pp. 1484$1495,2014$.

[8] "IEEE guide for synchronization, calibration, testing, and installation of Phasor Measurement Units (PMUs) for power system protection and control, IEEE Std C37.242 ${ }^{\mathrm{TM}}-2013$," IEEE Power and Energy Society, NY, USA, 2013.

[9] K. V. Khandeparkar, S. A. Soman, and G. Gajjar, "Detection and correction of systematic errors in instrument transformers along with line parameter estimation using PMU data," IEEE Trans. Power Systems, vol. 32, no. 4, pp. 3089-3098, 2017.

[10] A. Pal, P. Chatterjee, J. S. Thorp, and V. A. Centeno, "Online calibration of voltage transformers using synchrophasor measurements," IEEE Trans. Power Delivery, vol. 31, no. 1, pp. 370-380, 2016.

[11] R. F. Nuqui, M. Zarghami, and M. Mendik, "The impact of optical current and voltage sensors on phasor measurements and applications," in IEEE PES T\&D, New Orleans, 2010.

[12] S. Chakrabarti, E. Kyriakides, and M. Albu, "Uncertainty in power system state variables obtained through synchronized measurements," IEEE Trans. Instrum. Meas., vol. 58, no. 8, pp. 2452-2458, 2009.

[13] D. Roberson and J. F. O'Brien, "Loop shaping of a wide-area damping controller using HVDC," IEEE Trans. Power Systems, vol. 32, no. 3, pp. 2354-2361, 2017

[14] "IEEE standard for synchrophasor measurements for power systems, IEEE Std C37.118.1 ${ }^{\mathrm{TM}}-2011, "$ IEEE Power \& Energy Society, NY, USA, 2011.

[15] C. Huang, F. Li, D. Zhou, J. Guo, Z. Pan, and Y. Liu, "Data quality issues for synchrophasor applications Part I: a review," Journal of Mod. Power Syst. Clean Energy, vol. 4, no. 3, pp. 342-352, 2016.

[16] C. Huang, F. Li, T. Ding, Y. Jiang, J. Guo, and Y. Liu, "A bounded model of the communication delay for system integrity protection schemes," IEEE Trans. on Power Delivery, vol. 31, no. 4, pp. 1921-1933, 2016.

[17] S. Wang, X. Meng, and T. Chen, "Wide-area control of power systems through delayed network communication," IEEE Trans. Control Systems Technology, vol. 20, no. 2, pp. 495-503, 2012.

[18] F. Zhang, Y. Sun, L. Cheng, X. Li, J. H. Chow, and W. Zhao, "Measurement and modeling of delays in wide-area closed-loop control system," Trans. Power Systems, vol. 30, no. 5, pp. 2426-2433, 2015.

[19] A. Yogarathinam and N. R. Chaudhuri, "Wide-area damping control using multiple DFIG-based wind farms under stochastic data packet dropouts," IEEE Trans. Smart Grid, vol. 9, no. 4, pp. 3383 - 3393, 2016.

[20] T. Zabaiou, L.-A. Dessaint, F.-A. Okou, and R. Grondin, "Wide-area coordinating control of SVCs and synchronous generators with signal transmission delay compensation," in IEEE PES General Meeting, Minneapolis, 2010.

[21] F. Okou, L.-A. Dessaint, and O. Akhrif, "Power system stability enhancement using a wide area signals based hierarchical controller," IEEE Trans. Power Systems, vol. 20, no. 3, pp. 1465-1477, Aug. 2005.

[22] M. Asprou, S. Chakrabarti, and E. Kyriakides, "A two-stage state estimator for dynamic monitoring of power systems," IEEE Systems Journal, vol. 11, no. 3, pp. 1767-1776, 2017.

[23] M. Eremia and M. Shahidehpour, Handbook of Electrical Power System Dynamics, Wiley-IEEE Press, 2013.

[24] P. W. Sauer and M. A. Pai, Power System Dynamics and Stability, Prentice Hall, 1998.

[25] "Instrument transformers-Part 3: Additional requirements for inductive voltage transformers," IEC 61869-3:2011, 2011.

[26] "Instrument tranformers-Part 2: Additional requirements for current transformers," IEC 61869-2:2012, 2012. 
[27] "Model 1133A Power Sentinel GPS-Synchronized Power Quality/Revenue Standard, Operation Manual," Arbiter Systems Inc., Paso Robles, CA, USA, 2014.

[28] "IEEE standard for synchrophasor measurements for power systems, Amendment 1: Modification of selected performance requirements, IEEE Std C37.118.1 a ${ }^{\mathrm{TM}}-2014, "$ IEEE Power and Energy Society, NY, USA, 2014.

[29] ENTSO-E, "Analysis of CE inter-area oscillations of 1st December 2016," ENTSO-E, Brussels, 2017.

[30] "IEEE standard for synchrophasor data transfer for power systems, IEEE Std C37.118.2 $2^{\mathrm{TM}}-2011$," IEEE Power \& Energy Society, NY, USA, 2011.

[31] X. Zhang, C. Lu, X. Xie, and Z. Y. Dong, "Stability Analysis and Controller Design of a Wide-Area Time-Delay System Based on the Expectation Model Method," IEEE Trans.on Smart Grid, vol. 7, no. 1, pp. 520-529, 2016.

[32] Y. Wang, M. Haenggi, and Z. Tan, "The meta distribution of the SIR for cellular networks with power control," IEEE Trans. on Communications, vol. 66, no. 4, pp. 1745-1757, 2018.

[33] G. Huang, D. Akopian, and C. L. P. Chen, "Measurement and characterization of channel delays for broadband power line communications," IEEE Trans. on Instrumentation and Measurement, vol. 63, no. 11, pp. 2583-2590, 2014.

[34] B. P. Padhy, S. C. Srivastava, and N. K. Verma, "A wide-area damping controller considering network input and output delays and packet drop," IEEE Trans. Power Systems, vol. 32, no. 1, pp. 166-176, 2017.

[35] "IEEE guide for Phasor Data Concentrator requirements for power system protection, control, and monitoring, IEEE Std C37.244TM-2013," IEEE Power and Energy Society, NY, USA, 2013.

[36] B. P. Padhy, S. C. Srivastava, and N. K. Verma, "A network delay compensation technique for wide area SVC damping controller is power system," in IEEE PES T\&D Conference and Exposition, Chicago, 2014.

[37] L. Cheng, G. Chen, W. Gao, F. Zhang, and G. Li, "Adaptive time delay compensator (ATDC) design for wide-area power system stabilizer," IEEE Trans. on Smart Grid, vol. 5, no. 6, pp. 2957-2966, 2014.

[38] F. Zhang, L. Cheng, and W. Gao, "Prediction based hierarchical compensation for delays in wide-area control systems," IEEE Trans. on Smart Grid, vol. 9, no. 4, pp. 3897-3899, 2018.

[39] R. Liu, M. Mohanpurkar, M. Panwar, R. Hovsapian, A. Srivastava, and S. Suryanarayanan, "Geographically distributed real-time digital simulations using linear prediction," International Journal of Electrical Power \& Energy Systems, vol. 84, pp. 308-317, 2017.

[40] L. Zacharia, M. Asprou, and E. Kyriakides, "Design of a data delay compensation technique based on a linear predictor for wide-area measurements," in IEEE PES General Meeting, Boston, 2016. 